Relative abundance and seasonality of two populations of Bottlenose dolphins (Tursiops truncatus) in the Guaymas, Sonora region, Gulf of California

\title{
Abundancia relativa y estacionalidad de dos poblaciones de toninas (Tursiops truncatus) en la región de Guaymas, Sonora, Golfo de California
}

\author{
Daniel Guevara-Aguirre ${ }^{1 *}$, Juan Pablo Gallo-Reynoso²
}

\author{
'Posgrado de Ciencias del Mar y Limnología, Universidad Nacional Autónoma de México. Circuito Exterior s/n. Ciudad Universitaria \\ 04510, Distrito federal, México. E mail: danyguevara47@hotmail.com (DGA) \\ ${ }^{2}$ Laboratorio de Ecofisiología, Centro de Investigación en Alimentación y Desarrollo Guaymas. Carretera al Varadero Nacional km \\ 6.6, Sonora, 85480, México. E mail: jpgallo@ciad.mx (JPG) \\ *Corresponding author \\ ${ }^{*}$ Corresponding author
}

Introduction: The study of the ecology of the bottlenose dolphins (Tursiops truncatus) in the Gulf of California has been based on the distribution and abundance of the coastal ecotype; therefore there are no comparative studies of the ecology and seasonality of coastal and oceanic ecotypes in the central coast of Sonora. The aim of this study is to determine if seasonal changes have an effect on the relative abundance of the number of herds and the number of individuals of the two ecotypes (coastal and ocean) that inhabit the Guaymas Basin in the Gulf of California.

Methods: 13 trips to the coast and 16 to the ocean area where the number of herds and individuals were registered during one year to determine their seasonality. Seasonal relative abundance was obtained by the number of dolphins/hour, and the number of herds/hour. To determine the seasonality of dolphins, first we obtained the seasonal abundance by dividing the number of herds and the number of individuals, and second we made a hypothesis tests, with the number of herds and number of individuals and obtained a likelihood ratio for each ecotype.

Results: The greater abundance of coastal dolphins was during spring (18.52 dolphins/hour) and for the oceanic ones it was in winter (51.43 dolphins/hour; Tables 1 and 3). The seasonal analysis determined that the expected herd counts could be similar while the individuals count will be different for each season (Coastal: herds $\mathrm{D}=4.92$ ( $P$ $=0.1781)$; individuals $\mathrm{D}=61.50(P<0.001)$; and for Oceanic: herds $\mathrm{D}=7.78(P=0.0501)$; individuals $\mathrm{D}=547.42(P$ $<0.001$; Tables 2 and 4). Seasonally coastal dolphins present a greater number of herds and fewer individuals than the oceanic dolphins with the exception of spring season in both cases (Tables 5 and 6).

Discussion and conclusions: Areas of high primary productivity are of great importance to marine mammals and due to that, their distribution and overall relative abundance obtained for coastal dolphins/hour is one of the highest that we have found reported for other coastal regions (Baumgartner et al. 2001; Bazúa and Delgado 2014). These results agree with the characteristics of both ecotypes of dolphins, the oceanic dolphins presenting larger groups; and the coastal dolphins having a larger number of herds, except in spring, this is probably due to the movement of the oceanic dolphin's prey towards the continental shelf.

Key words: Bottlenose dolphins; ecotypes; relative abundance; seasonality. 


\section{Introducción}

Los delfines de la especie Tursiops truncatus, comúnmente llamados tursiones, toninas o bufeos (Gallo-Reynoso y Rojas-Bracho 1985), son uno de los mamíferos marinos mejor estudiados en el mundo debido a su distribución cercana a la costa. Esta condición permite su relación con diversas actividades humanas de importancia comercial, como las pesquerías; presentan una distribución casi cosmopolita y se distribuyen en casi todos los océanos, con excepción de los polos (Rice 1998; Wells y Scott 2002).

En el Golfo de California se ha observado que la distribución de los mamíferos marinos se relaciona con los movimientos de grandes masas de aguas oceánicas que otorgan un sinnúmero de características oceanográficas y provocan una alta productividad planctónica, que a su vez favorece la presencia de estos organismos. Se sabe que su distribución se vincula principalmente con la distribución de sus presas (Gallo-Reynoso y Figueroa-Carranza 1998; Jaquet y Gendron 2002).

Se han descrito dos ecotipos para esta especie en el Golfo de California y en otras partes del mundo: un ecotipo costero y otro pelágico (Hersh y Duffield 1990; Segura et al. 2006; Vázquez-Castán et al. 2009). La distribución del ecotipo costero se relaciona con lugares de alta productividad primaria, como las desembocaduras de los ríos, las lagunas costeras y las zonas de surgencia, y presentan patrones de movimientos limitados (Connor et al. 2000; Quintana-Rizzo y Wells 2001; Gallo-Reynoso et al. 2006). En cambio, el ecotipo oceánico presenta movimientos menos restringidos y puede ser encontrado en muchas áreas productivas, pero también se ha observado que algunas poblaciones pueden residir en los alrededores de las islas oceánicas (Reyes 1991; Gallo-Reynoso y Figueroa-Carranza 1998; 2005); por ello, al parecer, sus movimientos se vinculan directamente con la disponibilidad de sus presas y pueden desplazarse grandes distancias en aguas oceánicas en busca de alimento o aprovechar las poblaciones de presas locales costeras, como la lisa (Mugil cephalus) y la macarela (Scomberomorus maculatus; Mead y Potter 1990; Hoelzel 1994; Martín et al. 2000).

El tamaño del grupo es otra diferencia de estos ecotipos; el costero presenta manadas pequeñas (de 3 a 14 individuos) en la mayoría de las regiones costeras (Vázquez-Castán et al. 2007; Vázquez-Castán et al. 2009), mientras que el ecotipo oceánico presenta manadas de mayor tamaño (de 30 a 500 individuos; Ballance 1992; Defran y Weller 1999).

En México, la mayoría de los estudios de abundancia y estacionalidad de las toninas se han llevado a cabo en la zona costera. Lo anterior se debe a la dificultad de trabajar con organismos pelágicos, ya que al habitar en espacios abiertos carentes de una fisiografía como la presencia de la costa, no permite un fácil encuentro con estos organismos. Por ello, a pesar de que se han realizado estudios sobre poblaciones silvestres en el Golfo de California, en el Océano Pacífico, en el Golfo de México y en el Mar Caribe, se conoce muy poco sobre la situación poblacional de estos delfines oceánicos (Ortega-Ortiz et al. 2004).

En el Golfo de México se ha realizado la mayor cantidad de estudios acerca de la ecología de las toninas, principalmente en los estados de Campeche, Quintana Roo, Tabasco, Veracruz y Yucatán (Gallo-Reynoso 1988; Heckel 1992; Escatel 1997; Delgado 2002; Bazúa y Delgado 2014). La Laguna de Términos, en Campeche, es la zona con más estudios acerca de la ecología de las toninas. Allí se ha registrado la mayor abundancia relativa en el Golfo de México (13.6 toninas/ hora; Bazúa y Delgado 2014). En estos estudios, se ha determinado que las manadas de toninas presentan una marcada estacionalidad. En la temporada climática de "lluvias", se ha registrado una mayor abundancia relativa (toninas/hora), y ha disminuido durante las temporadas climáticas de "sequía" y de "nortes". Dichos resultados son similares a los obtenidos en las costas de Sinaloa, 
donde se encontró menor número de avistamientos de manadas en la temporada de sequía que en la de lluvias. Sin embargo, durante la sequía, el número de individuos fue mayor que en la temporada de lluvias (Reza 2001). El dato anterior difiere de lo reportado por Ruíz (1995), quien determinó que la mayor densidad de toninas en Bahía Banderas se dio durante el invierno.

El objetivo de este trabajo es determinar la estacionalidad y la abundancia relativa de dos ecotipos de toninas: costeras y oceánicas. Ambas habitan en la Cuenca de Guaymas, en el Golfo de California.

\section{Metodología}

Zona de estudio. El muestreo de los dos ecotipos de toninas se realizó en dos zonas del Golfo de California (GC). Para las toninas costeras, el estudio se llevó a cabo en la Bahía de las Guásimas, localizada en los $27^{\circ} 50^{\prime}$ norte y $-110^{\circ} 35^{\prime}$ oeste (Figura 1). Se trata de una zona somera que habitualmente presenta gradientes hipersalinos, aunque en época de lluvias extremas pueden encontrarse gradientes hiposalinos (Gallo-Reynoso et al. 2006). La Bahía de las Guásimas y sus sistemas lagunares adyacentes se originan en la llanura deltaica del Río Yaqui.

La batimetría y la forma son variables. Geomorfológicamente, corresponde a un valle inundado y ocupa planicies costeras anchas y de bajo relieve (Chávez y Álvarez 2006). Esta área es una importante zona pesquera, donde se pesca intensiva y extensivamente los camarones (Litopenaeus stylirostris y L. vannamei), las jaibas (Callinectes bellicosus y C. arcuatus), la medusa bola de cañón (Stomolophus meleagris) y diversos peces costeros y de laguna (Aragón y García 2002; Carvalho-Saucedo et al. 2011).

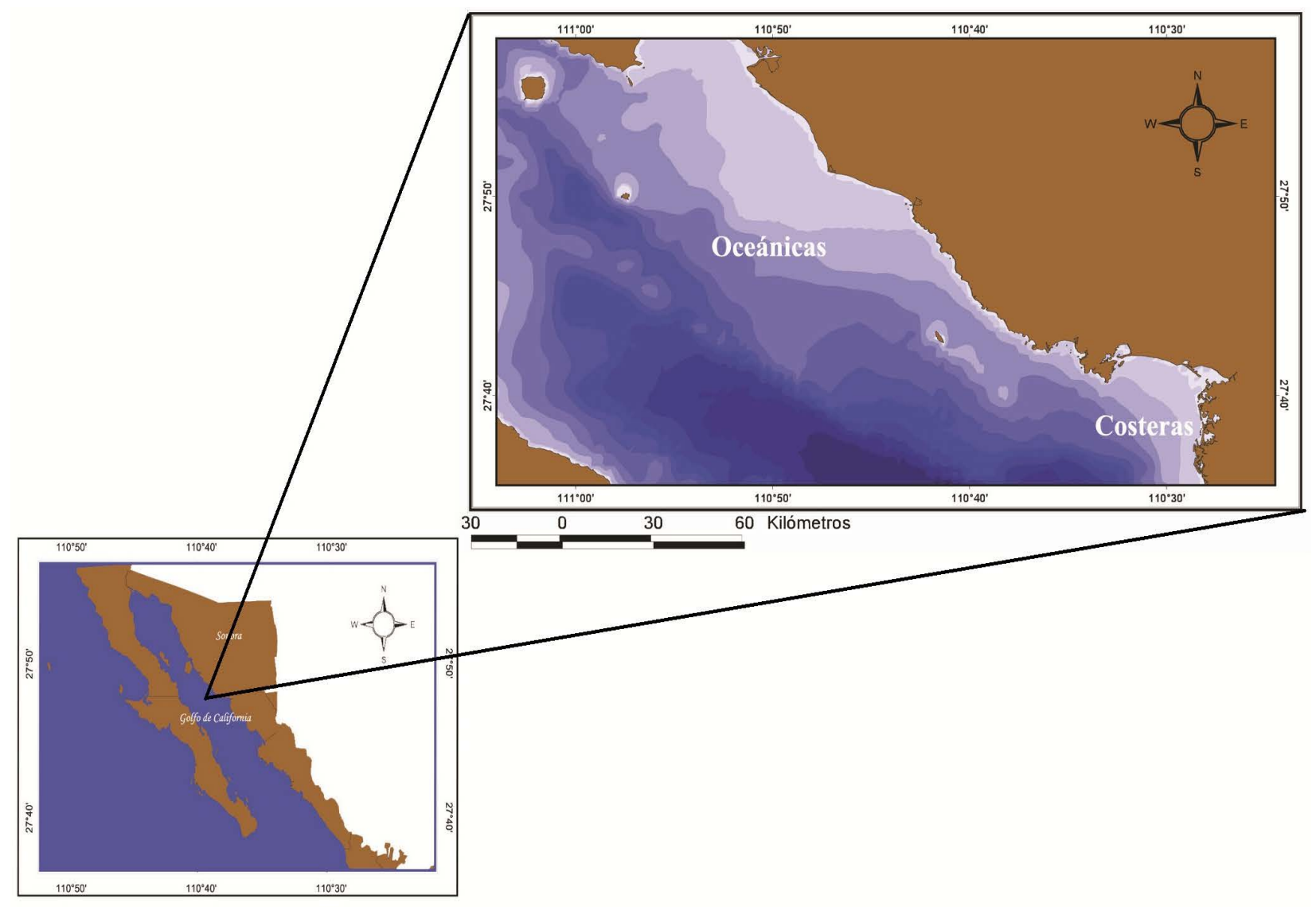

Figura 1. Área de estudio de los dos ecotipos de toninas (costeras y oceánicas). 
En cuanto a las toninas oceánicas, se muestreó la porción oeste de la costa central de Sonora, principalmente en el área de la Cuenca de Guaymas, en los alrededores de la Isla San Pedro Nolasco y en el espacio marítimo que va de la Isla San Pedro Nolasco a la Isla San Esteban. En esta área predomina un patrón estacional de vientos que influye en las corrientes superficiales. Durante el invierno, los vientos del noroeste producen surgencias muy fuertes en la zona costera continental, mientras que en el verano, los vientos que soplan del suroeste producen surgencias más débiles en la región costera peninsular (Roden 1964; Santamaría-Del-Ángel y ÁlvarezBorrego 1994). La batimetría de la zona corresponde a una gran porción de la Plataforma Costera, del Talud Continental y de los fondos asociados a las islas San Pedro Nolasco, San Pedro Mártir y San Esteban. En la zona de la Isla San Pedro Nolasco, se encuentra un bajo profundo (de 98 a 300 m) que hace las veces de dique de contención de las surgencias estacionales en el área. Por ello resulta un área de alta productividad en el invierno-primavera (Pérez y Sosa 2014).

Esfuerzo de búsqueda. Se realizaron 13 salidas de campo desde el poblado costero de Las Guásimas para muestrear a las toninas costeras. La primera salida fue en el otoño de 2012; la última, en el verano de 2013 (tres salidas en otoño de 2012, cuatro en invierno de 2013, tres en primavera de 2013 y tres en verano de 2013). Para estudiar a las toninas oceánicas, se realizaron 16 salidas de campo desde el poblado de La Manga, o desde San Carlos, hacia la Cuenca de Guaymas. La primera salida fue en la primavera de 2013; la última, en el invierno de 2014 (cuatro en primavera de 2013, cinco en verano de 2013, cuatro en otoño de 2013 y tres en invierno de 2014). Se cubrieron las cuatro estaciones climáticas que prevalecen en la zona. Los recorridos y la búsqueda de toninas en ambas zonas de estudio se realizaron en pangas de fibra de vidrio de 2022 pies de eslora con motor fuera de borda de 60-75 HP. Durante las salidas de campo de mayo, julio y septiembre (cuatro salidas), el recorrido se llevó a cabo en una embarcación tipo yate de 50 pies $(15 \mathrm{~m})$ de eslora.

Las embarcaciones fueron guiadas mediante un geo-posicionador y se controló la velocidad de desplazamiento. El objetivo primordial de las navegaciones fue la búsqueda específica de las manadas de toninas. No se usaron transectos lineales debido a la necesidad de obtener la localización geográfica precisa para caracterizar sus hábitats (i. e. la batimetría especifica del avistamiento). Dos o tres personas equipadas con binoculares con potencia de $10 \times 50$ realizaron las observaciones a una altura aproximada de dos a cuatro metros.

Una vez localizadas las toninas, se determinó un grupo focal, definido como un grupo de toninas en aparente asociación, que se mueve en una misma dirección y realiza la misma actividad (Shane 1990). El fenómeno anterior se registró en tablas previamente diseñadas y se añadió la posición geográfica de la embarcación (con un GPS), el número de avistamiento, la hora de inicio, la hora final del avistamiento y la hora a la que se interrumpía y reanudaba la búsqueda de manadas de toninas. La finalidad fue tomar en cuenta el tiempo real de búsqueda de toninas, a fin de considerar el esfuerzo realizado.

Abundancia relativa general. Para determinar la abundancia relativa general de los dos ecotipos de toninas, se realizó una estandarización de la abundancia al considerar al esfuerzo realizado, como el número de horas navegadas durante la búsqueda de toninas. Dicha ponderación del esfuerzo utilizó solamente el tiempo efectivo de navegación en actividad de búsqueda. Por ello, se eliminó el tiempo ocupado durante el seguimiento de las manadas y conteo de los individuos de toninas, así como de cualquier otra actividad no relacionada con la búsqueda de manadas. Para obtener el índice de la abundancia relativa general tanto de los individuos como de las manadas, se utilizaron las siguientes ecuaciones: índice de abundancia relativa = número de individuos de cada ecotipo / horas de esfuerzo y el índice de abundancia relativa = número de manadas de cada ecotipo / horas de esfuerzo. Dichas ecuaciones toman en cuenta la cantidad total de individuos y de manadas observadas durante el estudio para cada ecotipo de tonina. 
Adicionalmente se generaron mapas, con la distribución Kernel (en Arc-View 3.2) de ambos ecotipos de toninas para mostrar la densidad de uso, y de esta manera conocer las áreas más utilizadas por las toninas.

Estacionalidad. Con objeto de determinar la estacionalidad en las dos poblaciones de toninas, primero se obtuvo la abundancia relativa por estación climática para el número de manadas y de individuos; posteriormente, se realizó una prueba de hipótesis y se les aplicó una prueba de razón de verosimilitud (Casella y Berger 2001) con el paquete estadístico R, con el fin de determinar diferencias en el número de manadas y de individuos durante las cuatro estaciones climáticas de las zonas de estudio. A fin de determinar las diferencias estacionales entre ecotipos en el número de manadas y de individuos, se utilizaron los intervalos de confianza de $95 \%$ obtenidos por la prueba de razón de verosimilitud. Es importante mencionar que, si al comparar los intervalos de confianza, los valores no se interceptan, entonces podrá rechazarse que el conteo esperado de las manadas y de los individuos de ambos ecotipos sea el mismo. Por el contrario, si los intervalos de confianza se interceptan, entonces lo anterior indicaría que el conteo de manadas y de individuos de las toninas de ambos ecotipos son iguales.

\section{Resultados}

Esfuerzo de búsqueda. Durante las 13 salidas de campo para encontrar a las toninas costeras, se realizó un esfuerzo de búsqueda efectiva de 63.98 horas, en las que se observaron 74 manadas con 452 toninas. En cambio, en las 16 salidas de campo para encontrar a las toninas oceánicas, se registraron 95.37 horas efectivas de búsqueda de toninas, en las que se observaron 2,183 individuos agrupados en 45 manadas.

Distribución. La mayor densidad Kernel de los 74 avistamientos de manadas de toninas costeras se da en dos zonas: una ubicada en la boca de la Bahía de las Guásimas; la otra, en la boca del estero Los Algodones. En esta última zona, la densidad de avistamientos de manadas de toninas es mucho mayor que en la boca de la Bahía (Figura 2a). En contraste, respecto de las toninas oceánicas, pueden observarse dos zonas de mayor densidad kernel de avistamientos: una se encuentra entre la isla San Pedro Mártir y la isla San Esteban; la otra, alrededor de la isla San Pedro Nolasco. La máxima densidad se da hacia la parte sur de la isla, donde se halla una zona conocida como "el bajo", que es un bajo profundo (98 a 300 m; Figura 2b).

Abundancia relativa. La abundancia relativa general para los dos ecotipos de toninas nos indica que existe una mayor abundancia de toninas para el ecotipo oceánico (22.89 toninas/hora) que para el costero (12.27 toninas/hora). Sin embargo, el costero presenta una mayor abundancia relativa de manadas (2.00 manadas/hora) que el oceánico (0.47 manadas/hora).

Estacionalidad de las toninas costeras. Al determinar la estacionalidad como abundancia relativa (individuos/hora y manadas/hora), en el caso de las toninas costeras, la primavera fue la temporada climática con mayor abundancia relativa, seguida del otoño y del invierno. El verano fue la temporada climática con menor abundancia relativa. En relación con el número de manadas, se observó que los valores de otoño y de primavera fueron los más altos, seguidos del invierno. El verano fue también la temporada con menor número de manadas por hora. El esfuerzo de búsqueda fue muy similar para todas las estaciones climáticas. El invierno de 2013 fue la temporada en que se realizó un mayor esfuerzo de búsqueda. Lo anterior se debió a que en esta temporada se realizaron cuatro salidas de campo (Tabla1).

Se realizó una prueba de hipótesis sobre la igualdad del número esperado de conteos de manadas y de individuos por estación climática. En el caso de las manadas costeras, la estadística de prueba de la razón de verosimilitud fue $D=4.92(P=0.1781)$. En otras palabras, no es posible rechazar con $95 \%$ de confianza que el conteo esperado de manadas sea el mismo para cada estación climática. 
En el caso del número de individuos, la estadística de prueba fue $D=61.50(P \leq 0.001)$, i. e. Se rechaza con $95 \%$ de confianza que el conteo esperado de individuos en la zona costera sea el mismo para cada estación climática; se encontró un menor número de individuos durante el verano (Tabla 2). Estacionalidad de las toninas oceánicas. La abundancia relativa de las toninas oceánicas indica que la temporada climática que mayor abundancia de individuos presentó fue el invierno, seguido del otoño y el verano. La primavera fue la estación con la abundancia más baja. En relación con el número de manadas, el otoño fue la estación con mayor abundancia; le siguen el invierno y el verano. Finalmente, la primavera fue la estación en que se realizó el mayor esfuerzo de búsqueda (34.83 h), y el invierno la estación con menor esfuerzo de búsqueda (13.24 h). A pesar de que el verano tuvo la mayor cantidad de individuos observados (723), no fue la estación con mayor índice de abundancia relativa (Tabla 3).

Tabla 1. Número de individuos, número de mandas, I.A.R. o índice de abundancia relativa (toninas/hora) y horas de esfuerzo de las toninas costeras en la Bahía de las Guásimas, Sonora. Los valores en negritas cursivas corresponden a los valores más altos.

\begin{tabular}{|c|c|c|c|c|c|}
\hline \multicolumn{6}{|c|}{ Abundancia relativa de toninas costeras } \\
\hline Estación & $\begin{array}{c}\# \\
\text { Individuos }\end{array}$ & $\begin{array}{c}\# \\
\text { manadas }\end{array}$ & I.A.R. (individuos) & I.A.R. (manadas) & Esfuerzo(horas) \\
\hline Otoño 2012 & 130 & 23 & 15.00 & 2.65 & $8: 40$ \\
\hline Invierno 2013 & 108 & 19 & 10.56 & 1.85 & 10:13 \\
\hline Primavera 2013 & 163 & 21 & 18.52 & 2.38 & $8: 48$ \\
\hline Verano 2013 & 51 & 11 & 5.59 & 1.20 & 9:08 \\
\hline
\end{tabular}

Se realizó una prueba de hipótesis sobre la igualdad del número esperado de conteos de manadas y de individuos por estación climática (Tabla 4). En el caso de las manadas oceánicas, la estadística de prueba de la razón de verosimilitud fue $\mathrm{D}=7.78(P=0.0501)$ i. e. No es posible rechazar con $95 \%$ de confianza que el conteo esperado de manadas sea el mismo para cada estación climática.

En cuanto al número de individuos, la estadística de prueba de la razón de verosimilitud fue de $\mathrm{D}=547.42(P \leq 0.001)$, i. e. Se rechaza con $95 \%$ de confianza que el conteo esperado de individuos en la zona oceánica sea el mismo para cada estación climática. La primavera fue la estación con un menor número de individuos respecto de las otras tres estaciones climáticas (verano, otoño e invierno).

Comparación de las toninas costeras con las toninas oceánicas. Para realizar la comparación en la igualdad del número esperado de conteos de manadas durante las cuatro estaciones climáticas entre las toninas costeras y las oceánicas, se realizó una prueba de hipótesis. La estadística de prueba de la razón de verosimilitud fue $D=7.14(P=0.0075)$, i. e. Se rechaza con $95 \%$ de confianza que el conteo esperado de manadas en la zona costera sea el mismo que en la zona oceánica. De hecho, se espera un menor conteo de manadas oceánicas durante las cuatro estaciones climáticas. En la Tabla 5 se observa que en casi todas las estaciones climáticas, los intervalos se interceptan, excepto en la primavera, por lo que para esta estación se rechaza con una confianza de $95 \%$ que el conteo esperado de manadas en primavera sea el mismo para los ecotipos costeros y oceánicos; de tal forma, se espera un menor número de manadas oceánicas.

En relación con la comparación en la igualdad del número esperado de conteos de individuos costeros y oceánicos durante las cuatro estaciones climáticas, la prueba de hipótesis rechaza contundentemente $(P \leq 0.001)$ que el conteo esperado de individuos en la zona costera sea el 
mismo que en la zona oceánica. Asimismo, se espera un mayor conteo de individuos en la zona oceánica durante las cuatro estaciones climáticas. Solo en tres de las cuatro estaciones climáticas, los intervalos no se interceptan, excepto para la estación de primavera. Con una confianza de $95 \%$, se rechaza que el conteo esperado de individuos para las estaciones de otoño, invierno y verano sea el mismo para los ecotipos costero y oceánico; de tal forma, se espera un mayor conteo de individuos en la zona oceánica (Tabla 6). Por el contrario, en la primavera se espera que el conteo de individuos sea el mismo para ambos ecotipos.

a)

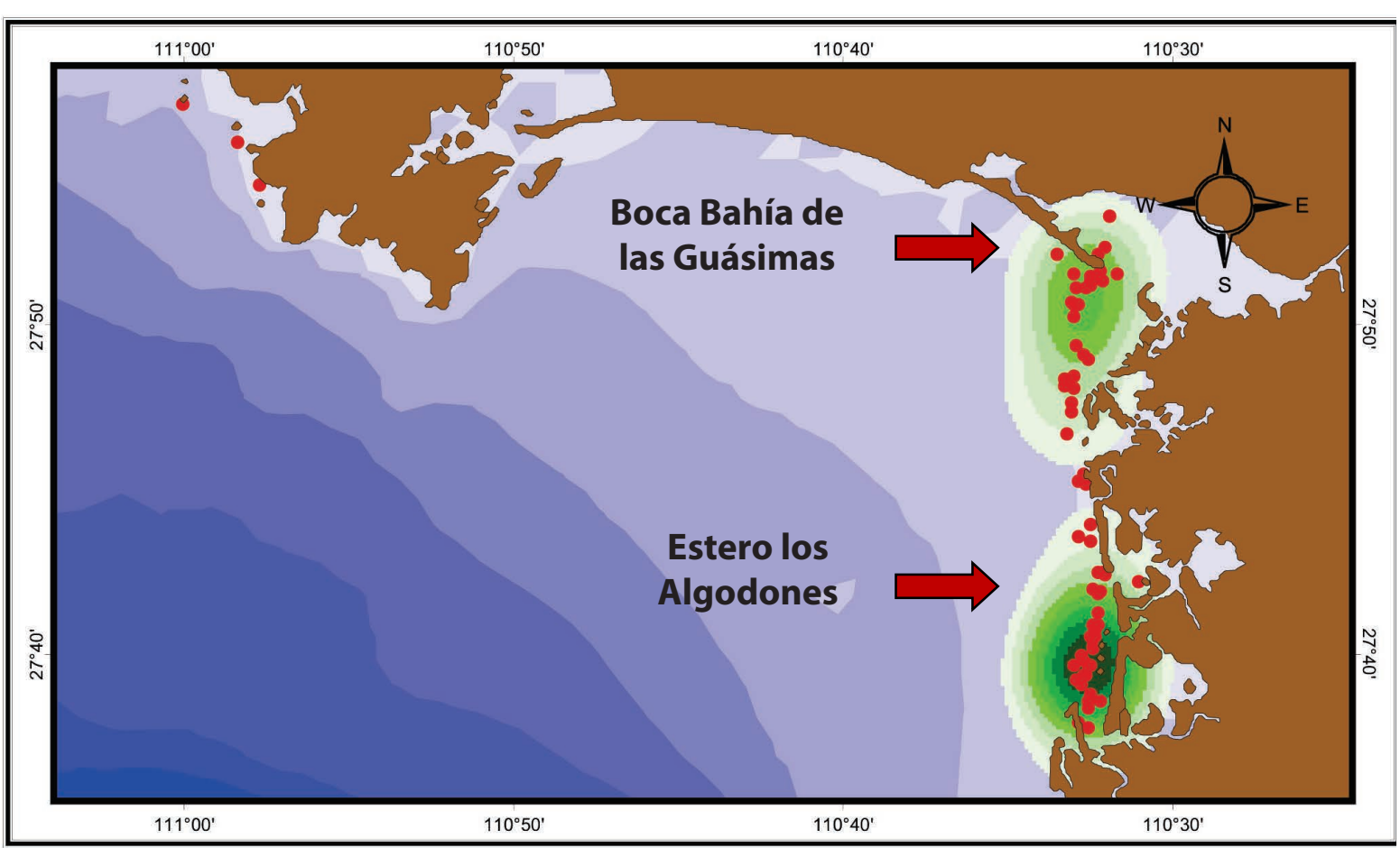

b)

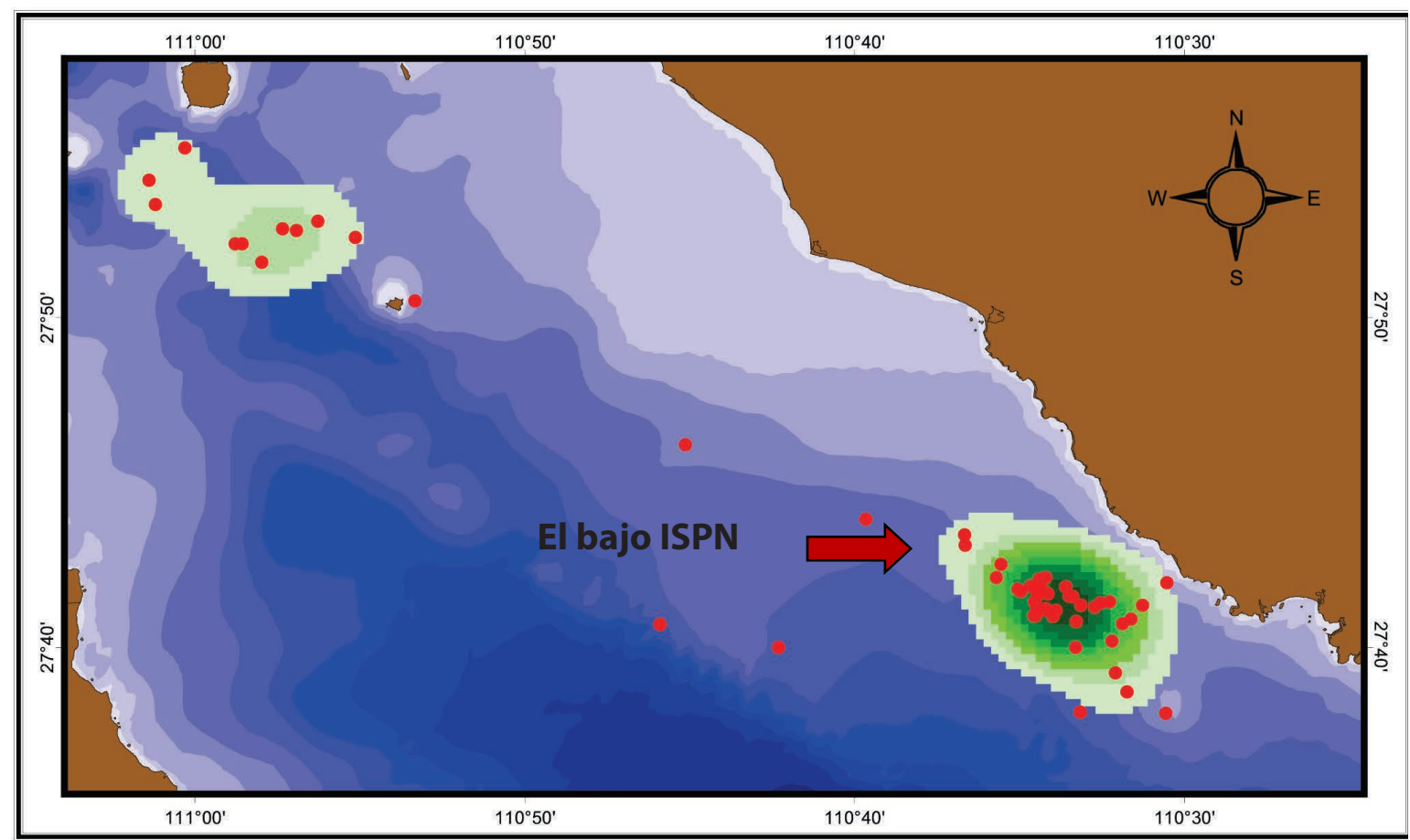

Figura 2. Mapas con la distribución Kernel de las dos poblaciones de toninas costeras y oceánicas de la región de Sonora. 
Tabla 2. Intervalos de confianza de $95 \%$ para el conteo esperado de manadas y de individuos de toninas costeras para las cuatro estaciones climáticas. En negritas cursivas, se muestra la estación que fue diferente a las demás, i. e. el verano.

\begin{tabular}{lcccc}
\multicolumn{1}{c}{ Estación } & \multicolumn{2}{c}{ Manadas } & \multicolumn{2}{c}{ Individuos } \\
\multicolumn{1}{c}{ climática } & Límite Inferior & Límite Superior & Límite Inferior & Límite Superior \\
\hline Otoño & 14.84098 & 33.71072 & 108.9323 & 153.6233 \\
Invierno & 11.69175 & 28.85900 & 88.90716 & 129.64826 \\
Primavera & 13.25633 & 31.29493 & 139.2606 & 189.2952 \\
Verano & 5.715521 & 18.831063 & $\mathbf{3 8 . 2 6 3 4}$ & $\mathbf{6 6 . 2 9 0 9}$ \\
\hline
\end{tabular}

\section{Discusión}

El Golfo de California es uno de los ecosistemas más biodiversos, dinámicos y productivos del planeta, ya que presenta tres mecanismos principales de fertilización: las surgencias inducidas por la batimetría y por el viento, la mezcla producida por los cambios semidiurnos de las mareas y la circulación termohalina. Lo anterior hace de este golfo una región de gran complejidad ecológica (Álvarez-Borrego 2002). Las zonas de alta productividad primaria tienen gran importancia para los mamíferos marinos. Se sabe que la mayoría de sus movimientos se relacionan con la presencia y distribución de sus presas y con los lugares de alta actividad biológica, como las descargas de ríos, las zonas estuarinas y las surgencias (Baumgartner et al. 2001).

Debido a dicha productividad, la abundancia relativa general obtenida en este estudio para el número de toninas costeras/hora es una de las más altas en comparación con las que se han encontrado en otras regiones costeras del Golfo de México. Incluso resultan muy similares a los de la Laguna de Términos (Bazúa y Delgado 2014), considerado como un lugar de alta actividad, gran diversidad y alta abundancia biológica (Ayala-Pérez 2003).

Distribución. El análisis de densidad Kernel nos indica que la densidad de manadas de toninas costeras se observó tanto en la boca de la Bahía de las Guásimas como en la boca del estero Los Algodones. Este último fue el de mayor densidad, posiblemente debido a que en dicha zona se encuentra la desembocadura de un ramal del Río Yaqui. Al mezclarse con el agua salada del golfo, genera un ecosistema con una elevada productividad biológica, como se ha observado en algunos estudios en el Golfo de México (Vázquez-Castán et al. 2009; Valdes-Arellanes et al. 2011).

En contraste, la mayor densidad de toninas oceánicas se presentó en la parte sur de la isla San Pedro Nolasco, donde se encuentra el bajo profundo que posiblemente, junto con la isla, sean causantes de una surgencia física que lleva agua rica en nutrientes a toda la columna de agua, y que es aprovechada por las potenciales presas de las toninas (De la Lanza 2004; COBI 2014; Gallo-Reynoso com. pers.). El fenómeno anterior influye de manera indirecta en la distribución potencial de las toninas, ya que algunos autores (Jaquet y Gendron 2002; Guevara-Aguirre 2011) han observado que la distribución de las toninas se relaciona directamente con la distribución de sus presas y con la fisiografía de la zona (tipo de fondo y de relieves).

Abundancia relativa. El ecotipo oceánico presentó una mayor abundancia relativa general en el número de individuos, pero una menor abundancia relativa en el número de manadas en comparación con el ecotipo costero. Esto puede explicarse por el tipo de asociación de ambos ecotipos. Encontramos que las manadas de toninas costeras son más pequeñas en promedio (6.1 toninas/manada) que las manadas de toninas oceánicas, (48.5 toninas/manada). El dato anterior es similar a lo que se ha encontrado en otros estudios (Ballance 1992; Defran y Weller 1999; Segura et al. 2006). 
Tabla 3. Índice de Abundancia Relativa (I.A.R. toninas/hora) de las toninas oceánicas. Los datos en negritas cursivas indican los valores más altos encontrados.

\begin{tabular}{|c|c|c|c|c|c|}
\hline \multicolumn{6}{|c|}{ Abundancia relativa de toninas oceánicas } \\
\hline Estación & \# individuos & \# manadas & I.A.R (individuos) & I.A.R (manadas) & Esfuerzo (horas) \\
\hline Primavera 2013 & 134 & 5 & 3.84 & 0.14 & $34: 50$ \\
\hline Verano 2013 & 723 & 16 & 28.05 & 0.62 & $25: 46$ \\
\hline Otoño 2013 & 645 & 15 & 30.39 & 0.71 & $21: 22$ \\
\hline Invierno 2014 & 681 & 9 & 51.43 & 0.70 & $13: 24$ \\
\hline
\end{tabular}

En cuanto al encuentro del ecotipo oceánico, se requirió realizar un mayor esfuerzo de búsqueda (en una proporción de 3:1; oceánicas $=95: 22 \mathrm{~h}$, costeras $=36.49$ h), debido a que las toninas oceánicas no se asocian a estructuras geomorfológicas o son residentes de algún área en específico. De tal modo, se incrementa el área donde se distribuyen; por lo tanto, se incrementa el tiempo de esfuerzo de su búsqueda.

Estacionalidad. En este estudio se observó una estacionalidad para ambos ecotipos, pero solo si se tomaba en cuenta el número de individuos y no el número de manadas. Lo anterior significa que durante todo el año de muestreo el número de manadas en las dos áreas de estudio se mantiene relativamente constante. Los resultados indican una estacionalidad en el número de individuos costeros. Allí se observa una similitud entre las estaciones climáticas de primavera, otoño e invierno. En cambio, el verano presenta la abundancia relativa más baja.

Estos resultados son diferentes de los reportados por Escatel (1997), Delgado (2002) y Bazúa y Delgado (2014), quienes encontraron una mayor abundancia relativa en la temporada de lluvias (verano). Lo anterior posiblemente se deba a que la laguna costera de las Guásimas recibe escasos aportes de agua dulce, lo que produce gradientes muy altos de salinidad que actúa principalmente como un anti-estuario (Gallo-Reynoso et al. 2006). Todo esto contrasta con lo observado en los estados del sur de México, donde en la temporada de lluvias, los numerosos ríos aportan cantidades importantes de nutrientes que causan alta productividad primaria en los sistemas lagunares.

Asimismo, durante el verano los vientos que soplan en el Golfo de California provienen del sureste y generan la entrada de una masa de agua del Pacífico nororiental, que es oligotrófica y de mayor temperatura (Bray y Robles 1991). Dicho fenómeno produce un decremento en la productividad primaria con aguas más claras, a diferencia de la primavera y el invierno, cuando se presentan las surgencias más fuertes en el lado continental y un incremento de la productividad primaria (Lara-Lara et al. 2008).

Tabla 4. Intervalos de confianza de $95 \%$ para el conteo esperado de manadas y de individuos de toninas oceánicas para las cuatro estaciones climáticas. En negritas cursivas se observa la estación climática que fue diferente a las demás, i. e. la primavera.

\begin{tabular}{lllll} 
Estación climática & $\begin{array}{l}\text { Manadas } \\
\text { Límite Inferior }\end{array}$ & Límite Superior & Límite Inferior & Límite Superior \\
\hline Otoño & 8.636459 & 23.912732 & 596.5372 & 696.0191 \\
Invierno & 4.327783 & 16.216664 & 631.1683 & 733.3879 \\
Primavera & 1.794911 & 10.739997 & $\mathbf{1 1 2 . 5 9 1 7}$ & $\mathbf{1 5 7 . 9 6 3 9}$ \\
Verano & 9.389673 & 25.159949 & 671.6163 & 776.9399 \\
\hline
\end{tabular}


En el caso de las toninas oceánicas, es posible que la menor abundancia relativa registrada durante la primavera, a pesar de ser la estación con mayor esfuerzo de búsqueda, se deba a que durante dicha estación climática las toninas oceánicas no se encuentran en los alrededores de la Isla San Pedro Nolasco, sino que se acercan a la plataforma continental, lo que probablemente se deba a que el ecotipo oceánico tiene un nivel trófico muy similar al de los cachalotes (Díaz-Gamboa 2003) y durante el mes de primavera (marzo y abril) los calamares gigantes (Dosidicus gigas) se mueven del centro del golfo hacia las zonas costeras, en donde durante mayo alcanzan tallas de entre 35 y $60 \mathrm{~cm}$ (Ehrhardt et al. 1986), por lo que las toninas posiblemente siguen los movimientos de los cachalotes y de la concentración de calamares.

Tabla 5. Intervalos de confianza de $95 \%$ para el conteo esperado de manadas por estación climática. Los valores en negritas cursivas indican diferencia en la comparación de las manadas costeras y oceánicas.

\begin{tabular}{lcccc} 
Estación & \multicolumn{2}{c}{ Manadas costeras } & \multicolumn{2}{c}{ Manadas oceánicas } \\
\multicolumn{1}{c}{ climática } & Límite Inferior & Límite Superior & Límite Inferior & Límite Superior \\
\hline Otoño & 14.84098 & 33.71072 & 8.636459 & 23.912732 \\
Invierno & 11.69175 & 28.85900 & 4.327783 & 16.216664 \\
Primavera & $\mathbf{1 3 . 2 5 6 3 3}$ & $\mathbf{3 1 . 2 9 4 9 3}$ & $\mathbf{1 . 7 9 4 9 1 1}$ & $\mathbf{1 0 . 7 3 9 9 9 7}$ \\
Verano & 5.715521 & 18.831063 & 9.389673 & 25.159949 \\
\hline
\end{tabular}

Al comparar los valores de número de manadas y número de individuos de los dos ecotipos por estación climática, observamos que en la primavera el número esperado de conteos de manadas costeras fue mayor que el de las oceánicas. No obstante, en términos de individuos, no existe diferencia estadística significativa. Por otro lado, en las otras estaciones climáticas (otoño, invierno y verano), no hay diferencias estadísticas en el número esperado de conteos de manadas en zonas costeras y oceánicas. Sin embargo, existe diferencia estadística en el número de individuos, que siempre fue mayor en oceánicas.

La estación climática de primavera se comporta de manera atípica. Se ha reportado (Ballance 1992; Defran y Weller 1999) que las manadas oceánicas son más grandes que las costeras, como sucedió en otoño, invierno y verano, debido quizá a las diferencias en el tipo de alimentación y a la manera de captura de sus presas. Las toninas oceánicas, al no contar con un sustrato para acorralar a sus presas, necesitan un mayor número de individuos que permitan encerrarlas. Por consiguiente, estas manadas han desarrollado una forma de captura cooperativa: acorralan a las presas en la superficie. De tal modo, el número de individuos que se necesitan es mucho mayor. Así disminuye el esfuerzo realizado y se incrementa la posibilidad de captura (Würsig y Würsig 1979).

Tabla 6. Intervalos de confianza de $95 \%$ para el conteo esperado de individuos por estación climática. Los valores en negritas cursivas indican las diferencias en la comparación estacional de los individuos de toninas costeras y oceánicas.

\begin{tabular}{lcccc}
\multirow{2}{*}{ Estación climática } & \multicolumn{2}{c}{ Individuos costeros } & \multicolumn{2}{c}{ Individuos oceánicos } \\
& Límite Inferior & Límite Superior & Límite Inferior & Límite Superior \\
\hline Otoño & $\mathbf{1 0 8 . 9 3 2 3}$ & $\mathbf{1 5 3 . 6 2 3 3}$ & $\mathbf{5 9 6 . 5 3 7 2}$ & $\mathbf{6 9 6 . 0 1 9 1}$ \\
Invierno & $\mathbf{8 8 . 9 0 7 1}$ & $\mathbf{1 2 9 . 6 4 8 2}$ & $\mathbf{6 3 1 . 1 6 8 3}$ & $\mathbf{7 3 3 . 3 8 7 9}$ \\
Primavera & 139.2606 & 189.2952 & 112.5917 & 157.9639 \\
Verano & $\mathbf{3 8 . 2 6 3 4}$ & $\mathbf{6 6 . 2 9 0 9}$ & $\mathbf{6 7 1 . 6 1 6 3}$ & $\mathbf{7 7 6 . 9 3 9 9}$ \\
\hline
\end{tabular}




\section{Conclusiones}

La distribución de los ecotipos de toninas (costeras y oceánicas) que habitan en la Cuenca de Guaymas se da en lugares que permiten alta productividad primaria; por ejemplo: la boca de la laguna de Guásimas y el estero Los Algodones, para las toninas costeras, y el sur de la isla San Pedro Nolasco sobre el bajo profundo. En relación con la estacionalidad, se observaron cambios temporales en ambos ecotipos, pero solo en el número de individuos y no en el de manadas. Las estaciones con menor número de individuos fueron el verano, para las toninas costeras, y la primavera, para las oceánicas.

Comparando estacionalmente ambos ecotipos en el número de manadas y en el número de toninas observadas durante la primavera, encontramos que esta se comportó de manera diferente de las otras tres estaciones climáticas. Presentó más manadas oceánicas que costeras y el mismo número de individuos en toninas costeras y en toninas oceánicas. En contraste, las otras estaciones (verano, otoño e invierno) presentaron más manadas en costeras que en oceánicas y menor número de individuos en toninas costeras que en toninas oceánicas.

\section{Agradecimientos}

Agradecemos al Posgrado de Ciencias del Mar y Limnología de la UNAM y al Centro de Investigación en Alimentación y Desarrollo A. C. por el espacio otorgado para trabajar los datos. A Comunidad y Biodiversidad (COBI) por el apoyo económico para la realización de este trabajo, al CONACYT por la beca otorgada a DGA; a J. Montoya por su valiosa ayuda en el análisis de los datos; a todos los integrantes del laboratorio de Ecofisiología del CIAD, por su valioso aporte en las salidas de campo y a J. L. Higuera de las Guásimas y a J. L. Ramírez de la Manga por su valioso apoyo y compañía.

\section{Resumen}

Introducción: El estudio de la ecología de las toninas (Tursiops truncatus) del Golfo de California se ha basado principalmente en la distribución y abundancia del ecotipo costero. No existen estudios comparativos sobre la ecología de los ecotipos costero y oceánico de la costa central de Sonora. El presente estudio pretende determinar la estacionalidad y la abundancia relativa de las manadas de los dos ecotipos de toninas que habitan en la Cuenca de Guaymas, en el Golfo de California.

Metodos: Se realizaron 13 salidas a la zona costera y 16 a la zona oceánica, y se utilizó un índice de abundancia relativa estacional.

Resultados: En el caso del ecotipo costero, la estación con mayor abundancia fue la primavera (18.52 toninas/ hora), y para el ecotipo oceánico fue el invierno (51.43 toninas/hora). Usando la estadística de prueba de la razón de verosimilitud, el análisis estacional por ecotipo determinó que no existen diferencias significativas en el conteo esperado de manadas para cada estación en ambos ecotipos; en cambio, el conteo de individuos será diferente para cada estación (Costeras: manadas: $\mathrm{D}=4.92(P=0.1781)$; individuos: $\mathrm{D}=61.50(P<0.001))$, (Oceánicas: manadas $\mathrm{D}=7.78(P=0.0501)$; individuos: $\mathrm{D}=547.42(P<0.001))$.

Discución y conclusiones: Al comparar estacionalmente a ambos ecotipos en el número de manadas y en el número de toninas observadas durante la primavera, encontramos que esta se comportó de manera diferente a las otras tres estaciones climáticas; presentó más manadas oceánicas que costeras y el mismo número de individuos en toninas costeras y en toninas oceánicas. Las otras estaciones (verano, otoño e invierno) presentaron más manadas en costeras que en oceánicas y menor número de individuos en toninas costeras que en toninas oceánicas.

Palabras clave: Abundancia relativa; ecotipos; estacionalidad, toninas.

\section{Literatura citada}

Álvarez-Borrego, S. 2002. Physical oceanography. Pp. 41-59 en New Island Biogeography of the Sea of Cortés (Case, T. J., M. L. Cody., y E. Ezcurra, eds.) Oxford University Press. Oxford, EE. UU. 
Aragón, N. E., y J. A. García. 2002. Incidencia de postlarvas de camarón (Crustacea: Penaidae) en el canal de llamada de una granja camaronera del estado de Sonora. Contribuciones al estudio de los crustáceos del Pacífico Este 1:145-154.

Ayala-Pérez, L. A., J. Ramos Miranda., y B. Gómez-Montes. 2003. La comunidad de peces de la Laguna de Términos: estructura actual comparada. Revista de Biología Tropical 51:783-794.

Ballance, L. T. 1992. Habitat use patterns and ranges of the bottlenose dolphin in the Gulf of California, Mexico. Marine Mammal Science 8:262-274.

Baumgartner, M. F., K. D. Mullin., L. N. May., y T. D. Leming. 2001. Cetacean habitats in the northern Gulf of Mexico. Fishery Bulletin 99:219-239.

Bazúa, D. C., y E. A. Delgado. 2014. Los tursiones, delfines de la laguna de Términos. México. Fomix Campeche 19:20-27.

Bray, N. A., Y J. M. Robles. 1991. Physical oceanography of the Gulf of California. Pp. 511-553 en The Gulf and Peninsular Province of the California (Douphin, J. P., y B. R. Simoneit, eds.). No. 47 American Association of Petroleum. Oklahoma. EE. UU.

Carvalho-Saucedo, L. J. López-Martínez, y F. García-Domínguez. 2011. Biología reproductiva de la medusa bola de cañón Stomolophus meleagris (Agassiz 1862) en la laguna Las Guásimas, Sonora, México. Hidrobiológica 21:77-88.

Chávez-López, S., y A. D. Álvarez-Arellano. 2006. Batimetría, sedimentos y ambientes de depósito en la laguna costera de Las Guásimas Sonora, México. Investigaciones Geográficas. UNAM. Boletín del Instituto de Geografía 60:7-21.

COBI. 2014. La reserva de la Biosfera Isla San Pedro Mártir. [en línea]: documento de fuente electrónica en internet. 2014 [fecha de consulta: 27 Abril 2014]. Disponible en: http://cobi.org.mx/wp-content/ uploads/2012/08/2007-d-0702_Folleto_ISPM_esp.pdf

Connor, R. C., R. S. Wells., J. Mann., y A. J. Read. 2000. The bottlenose dolphin. Social relationships in a fission-fusion society. Pp. 91-126 en Cetacean Societies. Field Studies of Dolphins and Whales (Mann, J, R. C. Connor, P. L. Tyack, y H. Whitehead, eds.). University of Chicago Press. Chicago, EE. UU.

De la Lanza-Espino, G. 2004. Gran escenario de la zona costera y oceánica de México. Ciencias 76:4-13.

Defran, R. H., y D. W. Weller. 1999. Occurrence, distribution, site fidelity, and school size of bottlenose dolphins (Tursiops truncatus) off San Diego, California. Marine Mammal Science 15:366-380.

Delgado, E. A. 2002. Comparación de parámetros poblacionales de las toninas, Tursiops truncatus, en la región sureste del Golfo de México (Estados Tabasco, Campeche, Yucatán y Quintana Roo). Tesis doctoral. Facultad de Ciencias, Universidad Nacional Autónoma de México. Ciudad de México, México.

Díaz-Gamboa, R. E. 2003. Diferenciación entre tursiones Tursiops truncatus costeros y oceánicos en el Golfo de California por medio de isótopos estables de carbono y Nitrógeno. Tesis de Maestría en Ciencias, CICIMAR. La Paz, México.

Ehrhardt, N. M., N. A. Solis, P. S. Jacquemin, C. J. Ortíz, R. P. Ulloa, D. G. González, y B. F. García. 1986. Análisis de la biología y condiciones del stock del calamar gigante Dosidicuos gigas en el Golfo de California, México, Durante 1980. Ciencia Pesquera 5:63-76.

Escatel Luna, R. E. 1997. Biología poblacional de las toninas Tursiops truncatus, en la Laguna de Términos, Campeche, México. Tesis de Maestría en Ciencias del Mar. Unidad Académica de los Ciclos profesionales y de Posgrado del Colegio de Ciencias y Humanidades, Universidad Nacional Autónoma de México. Ciudad de México, México.

Gallo-Reynoso, J. P., y L. Rojas Bracho. 1985. Nombres científicos y comunes de los mamíferos marinos de México. Anales del Instituto de Biología UNAM Serie Zoológica 56:1043-1056.

Gallo-Reynoso, J. P., y A. L. Figueroa-Carranza. 1998. Cetaceans of Isla de Guadalupe. Bulletin Southern California Academy of Sciences 97:33-38. 
Gallo Reynoso, J. P., y A. L. Figueroa Carranza. 2005. Los cetáceos de Isla Guadalupe. Pp. 202-217 en Isla Guadalupe: Restauración y Conservación (Santos del Prado, K., y E. Peters, eds.). Instituto Nacional de Ecología. Ciudad de México, México.

Gallo-Reynoso, J. P., J. ÉGido, E. Coria, y J. Saldaña. 2006. Distribución, Área de acción y población de toninas (Tursiops truncatus) en el área de la Bahía de las Guásimas, Sonora, México. Reporte interno del Centro de Investigación en Alimentación y Desarrollo, A.C., Unidad Guaymas. 45. Disponible en (available at) jpgallo@ciad.mx del autor por correspondencia.

Guevara-AguirRe, D. 2011. Relación de la presencia de toninas (Tursiops truncatus) en la Laguna de Términos, Campeche, México con los factores ambientales. Tesis de Maestría, Universidad Nacional Autónoma de México, PCMyL. Ciudad de México, México.

HeCKel, G. 1992. Foto-identificación de tursiones Tursiops truncatus (Montagu, 1821) en la Boca de Corazones de la Laguna de Tamiahua, Veracruz, México (Cetacea: Delphinidae). Tesis de Licenciatura en Biología, Facultad de Ciencias, Universidad Nacional Autónoma de México. Ciudad de México, México.

Hersh, S. L. y D. A. Duffield. 1990. Distinction between northwest Atlantic offshore and coastal bottlenose dolphins based on hemoglobin profile and morphometry. Pp. 129-139 en The Bottlenose Dolphin (Leatherwood, S., y R. R. Reeves, eds.) Academic Press. San Diego, EE. UU.

Hoezzel, A. R. 1994. Genetics and Ecology of whales and dolphins. Annual Review of Ecological Systems 25:377-399.

Jaquet, N., y D. Gendron. 2002. Distribution and relative abundance of sperm whales in relation to key environmental features, squid landings and the distribution of other cetacean species in the Gulf of California, México. Marine Biology 141:591-601.

lara Lara, J. R., V. Arenas Fuentes, M. D. C. Bazán, M. De la Cruz García, V. M. Díaz, E. Escobar, G. Gaxiola, E. G. Robles, R. Sosa, L. A. Soto, M. Tapia y J. E. Valdez. 2008. Los ecosistemas marinos. Pp.135-159 en Capital Natural de México (Soberón, J., G. Halffter., y J. Llorente, eds.) Vol. I: Conocimiento actual de la biodiversidad. Comisión Nacional para el Conocimiento y Uso de la Biodiversidad. Ciudad de México, México.

Martín, V., M. Carrillo, y L. F. López Jurado. 2000. The Bottlenose Dolphin in the Canary Islands. Gobierno de Canarias. Canarias, España.

Mead, J. G., Y CH. W. Potter. 1990. Natural history of bottlenose dolphins along the central Atlantic coast of the United States. Pp. 165-195 en The Bottlenose Dolphin (Leatherwood. S., y R. Reeves, eds.). Academic Press. San Diego, EE. UU.

Ortega-Ortiz, J. G., A. Delgado-Estrella, y A. Ortega-Argueta. 2004. Mamíferos marinos del Golfo de México, estado actual del conocimiento y recomendaciones para su conservación. Pp. 137-162 en Diagnóstico ambiental del Golfo de México (Caso, M., I. Pisanty, y E. Exequiel, eds.). Secretaría de Medio Ambiente y Recursos Naturales/ Instituto Nacional de Ecología/ Instituto de Ecología/Harte Research Institute for Gulf of Mexico Studies. Ciudad de México, México.

Quintana-Rizzo, E., y R. S. Wells. 2001. Resighting and association patterns of bottlenose dolphins (Tursiops truncatus) in the Cedar Keys, Florida: Insights into social organization. Canadian Journalof Zoology 79:447-456.

Pérez y Sosa, M. C. 2014. Distribución espacial de mamíferos marinos asociada a la geomorfología del fondo marino alrededor de la Isla San Pedro Nolasco en el Golfo de California. Tesis de maestría, Universidad Nacional Autónoma de México, PCMyL. Ciudad de México, México.

Reyes, J. C. 1991. The conservation of small cetaceans: a review. Report prepared for the Secretariat of the Convention on the Conservation of Migratory Species of Wild Animals. UNEP / CMS Secretariat. Bonn, Alemania.

Reza, N. I. 2001. Distribución y abundancia de Tursiops truncatus en la Bahía de Santa María, Sinaloa, México. Tesis profesional, Facultad de Ciencias, Universidad Nacional Autónoma de México. Ciudad de México, México. 
RICE, D. W. 1998. Marine mammals of the world. Systematics and distribution. Special Publication No. 4. The Society for Marine Mammalogy. Allen Press. Lawrence, EE. UU.

Roden, G. I. 1964. Oceanographic aspects of the Gulf of California. Pp. 30-58 en Marine Geology in the Gulf California (Van Andel., Tj. H., y. G. G. Shor, eds.) No. 3 American Association of Petrology Geologists. EE.UU.

Ruiz Boijseauneau, B. I. 1995. Distribución y abundancia de Tursiops truncatus Montagu, 1821 (Cetacea: Delphinidae) en la bahía de Banderas y aguas adyacentes de México. Tesis profesional, Facultad de Ciencias, Universidad Nacional Autónoma de México. Ciudad de México, México.

Santamaría-del-Ángel, E., y S. Álvarez-Borrego. 1994. Gulf of California biogeographic regions based on coastal zone color scanner imagery. Journal of Geophysical Research 99:7411-7421.

Segura, I. H., A. Rocha, S. Flores, y L. Rojas. 2006. Conservation implications of the genetic and ecological distinction of Tursiops truncatus ecotypes in the Gulf of California. Biological Conservation 133:336346.

Shane, S. H. 1990. Behavior and ecology of the bottlenose dolphin at Sanibel Island, Florida. Pp. 245265 en The bottlenose dolphin (Leatherwood, S., y R. R. Reeves. eds). Academic Press. San Diego.

Valdéz-Arellanes, M. P., A. Serrano, G. Heckel, Y. Schramm, e I. Martínez-Serrano. 2011. Abundancia de dos poblaciones de toninas (Tursiops truncatus) en el norte de Veracruz, México. Revista Mexicana de Biodiversidad 82:227-235.

Vázquez-Castán, L., A. Serrano, M. López-Ortega, J. A. Galindo, M. P. Valdéz-Arellanes, y C. Naval-Ávila. 2007. Caracterización del hábitat de dos poblaciones de toninas (Tursiops truncatus, Montagu 1821) en la costa norte del estado de Veracruz, México. Revista Científica UDO Agrícola 7:285-292.

Vázquez-Castán, L., A. Serrano, y J. A. Galindo. 2009. Estudio preliminar sobre la biodiversidad y abundancia de cetáceos en aguas profundas del Golfo de México. Revista UDO Agrícola 9:992997.

WürSIG, B., WüRSIG, M. 1979. Behavior and ecology of the bottlenose dolphin (Tursiops truncatus) in the south Atlantic. Fishery Bulletin 77:399-412.

Wells, R. y M. Scott. 2002. Bottlenose dolphins Tursiops truncatus and T. aduncus. Pp. 122-128 en Encyclopedia of Marine Mammals (Perrin, W., B. Würsig, y J. Thewissen, eds.). Academic Press. San Diego, EE. UU.

Summited: February 23, 2015

Review: March 2, 2015

Accepted: March 26, 2015

Associated editor: Sergio Solari 